\title{
Belal Batiha
}

\section{You paid \$200.00 USD}

to Belal Batiha

Details

Get PayPal Notifications in Messenger

\section{Paid with}

PayPal Credit

$\$ 200.00$ USD

This transaction will appear on your statement as SCIENCEPUB

\section{Purchase details}

Receipt number: 2DU76149EK0871432

We'll send confirmation to:

taleb.moazzeni@yahoo.com

\section{Merchant details}

Belal Batiha

\section{Return to Merchant}

Policies Terms Privacy Feedback @ $1999-2020$ 